\section{Beine hoch bei Anaphylaxie!}

\author{
Ein Leserbrief an den Herausgeber des Journal of Allergy and Clinical \\ Immunology mahnt die Grundlagen im Umgang mit anaphylak- \\ tischen Patienten an: Die Schocklagerung ist nach wie vor die erste \\ und entscheidende Maßnahme.
}

Ei in Pathologe aus Manchester dokumentiert seit dem Jahr 1992 Todesfälle durch anaphylaktischen Schock. Bei 196 Fällen lagen ausreichende Informationen vor, um die Todesursache festzustellen: Die meisten Patienten starben an Asphyxie (96 Fälle) oder Schock (88 Fälle, davon 38 mit Dyspnoe).

Bei zehn von 38 außerklinischen Todesfällen war dokumentiert, in welcher Körperlage sich die Patienten befanden, bevor sie starben. Vier der anaphylaktischen Patienten starben innerhalb von Sekunden, nachdem sie eine aufrechtere Position eingenommen hat- ten: Drei waren aufgestanden, einer setzte sich auf. Die anderen sechs Patienten waren sitzend gelagert worden, nachdem sie das Bewusstsein verloren hatten.

Der anaphylaktische Schock ist geprägt durch Vasodilatation und gestörte Gefäßpermeabilität. In der Horizontalen kann der venöse Rückfluss zum Herzen noch ausreichen, um einen minimalen Kreislauf aufrecht zu halten. Doch beim Aufrichten des Oberkörpers entleert sich die Vena cava innerhalb weniger Sekunden, die Ventrikelfüllung bleibt aus, elektromechanische Dissoziation und Myokardischämie folgen.
Sind die Vena cava einmal leer und der Kreislauf unterbrochen, bleiben Adrenalin sowie die Herzdruckmassage wirkungslos. Zuerst benötigt der Patient den Ausgleich des absoluten und relativen Volumenmangels durch Infusionen und Autotransfusion - also Beine hoch! Je größer der Volumenmangel, desto effektiver ist die Autotransfusion. Das habe eine aktuelle Studie gezeigt.

Fazit: Allergiekranke sollten im Falle einer anaphylaktischen Reaktion - außer bei Atemnot - liegen bleiben, auch bei Anwendung ihrer Notfallmedikamente. Eventuelle Ersthelfer (Familie, Lehrer, Freunde, Kollegen) müssen das einfache, aber lebensrettende „Beine hoch!“ kennen. Auch beim Transport muss jegliches Aufrichten unterbleiben.

Pumphrey RSH. Fatal posture in anaphylactic shock. J Allergy Clin Immunol 2003; 112: 451-2.

\title{
Die Rolle der Interleukine beim Schock
}

\section{Interleukin-4 (IL-4) und das nahe verwandte IL-13 sind entscheidend an der Allergieauslösung beteiligt. Der Verdacht liegt nahe, dass sie auch beim anaphylaktischen Schock eine wichtige Rolle spielen.}

L-4 fördert Allergien. Das Zytokin erhöht die IgE-Produktion, lockt Eosinophile an, aktiviert Mastzellen und unterdrückt die Produktion von antiallergischem IL-12. Die Arbeitsgruppe um Fred Finkelman von der University of Cincinnati wies jetzt auch eine wesentliche Rolle von IL-4 beim anaphylaktischen Schock nach.

Die Forscher lösten in Mäusen einen akuten Schockzustand aus, in dem sie IgE- (FceRI) oder auch IgG-Rezeptoren (Fc $\gamma$ RII/RIII) aktivierten (siehe Kasten). Daraufhin fiel die Körpertemperatur innerhalb von 60 Minuten von 38 bis auf ca. $30^{\circ} \mathrm{C}$ ab; doch danach erholten sich die Tiere wieder. Waren sie jedoch mit IL-4 vorbehandelt oder erzeugten sie erhöhte Mengen an IL-4 durch ein zusätzlich eingepflanztes IL4-Gen, so fiel ihre Körpertemperatur auf unter $25^{\circ} \mathrm{C}$ ab. Dies führte bei vielen Tieren direkt zum Tod.
Den Beweis für IL-4 als Schockverstärker lieferten Mäuse ohne IL-4Rezeptor (IL-4R $\alpha$ ), die sich vermindert schocken ließen. Ein zusätzlicher Schuss IL-12 oder IL-18 blockierte die Schockreaktion ebenfalls. Als Wirkungsweise von IL-4 fanden die Forscher eine Verstärkung der vasoaktiven Funktionen von Histamin und des Platelet Activating Factor (PAF), die zu erhöhter Gefäßpermeabilität führen. Glichen sie den abfallenden Blutdruck durch Volumenersatz aus, überlebten $80 \%$ der Mäuse den schweren Schockzustand.

Fazit: IL-4 (und IL-13) sind nicht nur maßgeblich an der Auslösung von Allergien beteiligt, sie spielen ebenfalls eine zentrale Rolle im akuten allergischen Schockgeschehen - zumindest bei Mäusen. Wahrscheinlich wirken die Zytokine im Verein mit Histamin und PAF direkt auf die Permeabilität der Gefäße. Die
Blockierung von IL-4 könnte demnach als eine pleiotrop wirksame Antischock therapie in Frage kommen, die sowohl die IgE- als auch die IgG-abhängige Schockreaktion unterdrückt.

Strait RT et al. IL-4 exacerbates anaphylaxis. J Immunol 2003; 170: 3835-42

Schock über IgE- und IgGRezeptoren

Nicht nur IgE führt über seinen Rezeptor (FcERI) und die bekannte Reaktionskaskade (Aktivierung von Mastzellen und Basophilen, Ausschüttung von Mediatoren) zum anaphylaktischen Schock. Die Arbeitsgruppe um Fred Finkelman konnte an Mäusen zeigen, dass Allergenspezifisches IgG über seinen Rezeptor Fc $\gamma$ RII/RIII zur Aktivierung von Makrophagen und zur Ausschüttung von PAF führt und mindestens ebenso stark wirkt. Als Modell verwendeten die Forscher die Aktivierung von $\mathrm{Fc \in RI}$ oder Fc $\gamma \mathrm{RII} / \mathrm{RIII}$ über die intravenöse Gabe von IgE- oder IgG-Antikörperkomplexen, die an die entsprechenden Rezeptoren binden.

Strait RT et al. Pathways of anaphylaxis in the mouse. J Allergy Clin Immunol 2002; 109: 658-68 